\title{
Evaluation of temporal moments and Fourier transformed data in time-domain diffuse optical tomography
}

\author{
Meghdoot Mozumder ${ }^{1, *}$ And TANJA TARVAinen ${ }^{1,2}$ \\ ${ }^{1}$ Department of Applied Physics, University of Eastern Finland, P.O. Box 1627, 70211 Kuopio, Finland \\ ${ }^{2}$ Department of Computer Science, University College London, Gower Street, London WC1E 6BT, United Kingdom \\ *Corresponding author: meghdoot.mozumder@uef.fi
}

Compiled January 14, 2021

\begin{abstract}
Time-domain diffuse optical tomography (TD-DOT) uses near infrared pulsed lasers as light sources and measure time-varying exitance on the boundary of the target. These are used to estimate optical properties of the imaged target. Several integral transform based moments of the time-resolved data have been utilised in TD-DOT, the most common being the mean time of flight and variance. Recently, it has been shown that Fourier transforming the time-domain data to frequency domain enables utilisation of this data at one or several frequencies, producing equally as good estimates as the whole time-domain data. In this work, we present a systematic comparison of the usage of the temporal moments and Fourier transformed data in TD-DOT. Both absolute and difference imaging are evaluated using numerical simulations. The simulations show that utilising temporal moments and Fourier transformed data in TD-DOT provides good quality reconstructions with a good estimation accuracy. These estimates are improved if more than one data type are used. Furthermore, the simulations show that the frequency domain computations enable computationally cheaper and straightforward implementation of the inverse solver when compared to the temporal moments. @ 2021 Optical Society of America
\end{abstract}

http://dx.doi.org/10.1364/ao.XX.XXXXXX

\section{INTRODUCTION}

Diffuse optical tomography is a technique for imaging spatially varying optical properties, typically the absorption and scattering parameters or quantities computed from these such as oxygen saturation, in biological tissues [1-3]. The distribution of these optical parameters provide tissue biochemical and structural information with applications for example in imaging breast cancer, monitoring treatments, brain imaging and small animal studies, see e.g. [3-6] and the references therein.

Time-domain diffuse optical tomography (TD-DOT) uses pulsed lasers for illuminating the tissues. The time-varying exitance, i.e. temporal point spread function (TPSF), is measured at detectors on the boundary of the target domain. The benefits of TD-DOT system include capability to image also through large thicknesses of tissue and the large information content of the measured TPSF [2, 7]. Several TD-DOT systems have been developed and applied e.g. in imaging tissue-mimicking phantoms [8,9], in brain studies [10-13], and in breast imaging [14, 15].

Various image reconstruction approaches have been proposed to estimate the spatially varying optical parameters us- ing TD-DOT. These include using the whole time-resolved data $[16,17]$, using integral transform temporal moments of the time-resolved data [18-22], Fourier transform based approaches $[23,24]$, and utilising different time-windows of the time-resolved data $[25,26]$. A short summary of data types utilised in some of the previous TD-DOT studies is given in Table 1.

Using the whole time-resolved data was shown to be the most accurate method in estimating the optical parameters in an earlier study [16]. However, using the whole time-resolved data required around $1100 \%$ higher computation time compared to using temporal moments [16]. Temporal moments considered in TD-DOT are, for example, the first temporal moments (or Mellin transforms) [20, 22, 35], Laplace transform [1,36] and the Mellin-Laplace transform $[1,21]$. Use of temporal moments has led to reduction of computation time and memory requirements due to compression of the time-resolved data [20].

Use of other temporal moments have also been proposed, such as an integral of the time-resolved data over a temporal period (time-gating), the peak intensity of the data and a logarithmic slope of the temporal decay [21]. Ref. [34] studied the use of different shapes of windows for time-gating in TD-DOT. 
Table 1. Data types used in TD-DOT, reconstructed parameters and applications in chronological order. The symbols used are: E: time integrated intensity, $<t>$ : first temporal moment (mean time of flight), $c_{2}$ : second central moment (variance), $c_{3}$ : third central moment, LT: Laplace transform. $\mu_{\mathrm{a}}$ : absorption coefficient, and $\mu_{\mathrm{s}}^{\prime}$ : reduced scattering coefficient.

\begin{tabular}{cccc}
\hline Ref. & Moment & Reconstructed parameters & Application \\
\hline$[8]$ & $\mathrm{E}$ & Difference $\mu_{\mathrm{a}}$ & Cylindrical phantom \\
{$[27]$} & $<t>, c_{2}, \mathrm{LT}$ & Difference and absolute $\mu_{\mathrm{a}}, \mu_{\mathrm{s}}^{\prime}$ & Cylindrical phantom \\
{$[10]$} & Absolute $\mu_{\mathrm{a}}, \mu_{\mathrm{s}}^{\prime}$ & Cylindrical phantom \\
{$[28]$} & Difference and absolute $\mu_{\mathrm{a}}, \mu_{\mathrm{s}}^{\prime}$ & Human forearm movement \\
{$[29]$} & $<t>c_{2}, \mathrm{LT}$ & Difference $\mu_{\mathrm{a}}, \mu_{\mathrm{s}}^{\prime}$ & Infant brain haemorrage \\
{$[16]$} & Whole data, $\mathrm{E},<t>, c_{2}, c_{3}$. & Absolute $\mu_{\mathrm{a}}, \mu_{\mathrm{s}}^{\prime}$ & Simulations \\
{$[23]$} & Fourier transform & Difference $\mu_{\mathrm{a}}, \mu_{\mathrm{s}}^{\prime}$ & Infant brain cortical response \\
{$[30]$} & Fourier transform & Absolute $\mu_{\mathrm{a}}, \mu_{\mathrm{s}}^{\prime}$ & Cylindrical phantom \\
{$[31]$} & $<t>$ & Difference $\mu_{\mathrm{a}}, \mu_{\mathrm{s}}^{\prime}$ & Infant brain oxygenation \\
{$[32]$} & Whole data & Difference $\mu_{\mathrm{a}}$ & Liquid phantom tank \\
{$[22]$} & $<t>$ & Absolute $\mu_{\mathrm{a}}$ & Cylindrical phantom \\
{$[26]$} & Mellin-Laplace transform & Absolute $\mu_{\mathrm{a}}, \mu_{\mathrm{s}}^{\prime}$ & Simulations \\
{$[17]$} & Whole data & Difference $\mu_{\mathrm{a}}, \mu_{\mathrm{s}}^{\prime}$ & Simulations \\
{$[33]$} & Mellin-Laplace transform & Difference $\mu_{\mathrm{a}}$ & Cuboid phantom \\
{$[15]$} & E & Absolute $\mu_{\mathrm{a}}$ & Breast cancer phantom \\
{$[34]$} & Temporal windows & Absolute $\mu_{\mathrm{a}}$ & Simulations \\
\hline
\end{tabular}

These data types partially use the full temporal information measured and a few other studies have reported their sensitivity to depth and resolution $[11,37,38]$. To our knowledge they have not been studied in detail and compared to the other moments for the TD-DOT reconstruction problem.

A comparison of the estimation accuracy obtained using different temporal moments, using one in silico target, was presented in [21]. It was shown that using only one moment was inadequate to reconstruct both absorption and scattering parameters simultaneously. It was suggested that this was due to non-uniqueness of the reconstruction problem, similarly as in using intensity data alone [39]. Using a combination of moments was shown to improve results. However, a systematic study of the number of the temporal moments required for a reasonable quality reconstruction has not been carried out. Ref [21] demonstrated that the choice of the optimal moment depends on the distribution of target optical properties, which is only partially known in real applications.

Time-domain and frequency-domain DOT have a natural relationship through a Fourier transform [1]. Approaches where TDDOT data have been transformed to frequency domain haven been utilised in DOT e.g. in [23, 24, 30, 40]. Fourier transformed time-resolved data at one frequency has been utilised e.g. in phantom studies $[30,40]$ and reconstructing optical parameter changes due to brain activation [23]. In a recent work, we numerically studied utilisation of multiple Fourier frequencies in TD-DOT [24]. The results showed that utilising the Fourier series at multiple frequencies resulted in better optical parameter estimates than using only one frequency, and that the accuracy of these estimates was comparable to using the whole timeresolved data. Furthermore, utilising the frequency domain data required significantly less computational time and resources than the reconstructions from the whole time-resolved data.

Although different data types for TD-DOT have been proposed and studied, their performance in the inverse problem of DOT has not been systematically studied. Neither, there has not been a thorough comparison of the number of data types needed to obtain reasonable good estimates for absorption and scattering.

In this paper, we compare different data types utilised in TDDOT by studying the inverse problem of DOT. The data types compared are the most often used temporal moments, i.e. first temporal moment and and second central moment and their combination, and utilising one or several frequencies of Fourier transformed data. The inverse problem for estimating absorption and scattering parameters are formulated for different data types. Both difference and absolute imaging are studied.

The rest of the paper is organized as follows. Modelling light transport in TD-DOT and computing different temporal moments and frequency domain data are reviewed in Sec. 2. Then, the inverse problem of TD-DOT utilising these data types is described in Sec. 3. The numerical simulations are described in Sec. 4 and the results are presented in Sec. 5, followed by discussion and conculsions in Sec. 6.

\section{MODEL FOR LIGHT PROPAGATION}

\section{A. Time-domain diffuse optical tomography}

In a typical TD-DOT measurement setup, near-infrared light is introduced into an object from its boundary. Let $\Omega \subset \mathbb{R}^{d}$, $(d=$ 2 or 3 ) denote the domain with boundary $\partial \Omega$ where $d$ is the (spatial) dimension of the domain. In a diffuse medium like biological tissue, the commonly used light transport model for 
TD-DOT is the diffusion approximation (DA) to the radiative transfer equation $[1,41]$

$$
\left\{\begin{array}{l}
\left(-\nabla \cdot \frac{1}{d\left(\mu_{\mathrm{a}}(r)+\mu_{\mathrm{S}}^{\prime}(r)\right)} \nabla+\mu_{\mathrm{a}}(r)+\frac{1}{c} \frac{\partial}{\partial t}\right) \Phi(r, t)=0, r \in \Omega \\
\Phi(r, t)+\frac{1}{2 \gamma_{d}} \frac{1}{d\left(\mu_{\mathrm{a}}(r)+\mu_{\mathrm{s}}^{\prime}(r)\right)} \alpha \frac{\partial \Phi(r, t)}{\partial \hat{n}}=\left\{\begin{array}{l}
\frac{Q(r, t)}{\gamma_{d}}, r \in s \\
0, r \in \partial \Omega \backslash s
\end{array}\right.
\end{array}\right.
$$

where $\Phi(r, t)$ is the photon fluence at a point $r$ and time instance $t, \mu_{\mathrm{a}}(r)$ is the absorption coefficient, and $\mu_{\mathrm{s}}^{\prime}(r)$ is the (reduced) scattering coefficient. Further, $c$ is the speed of light in the medium, $Q(r, t)$ is the pulsed (temporal) light source at boundary locations $s \subset \partial \Omega$. The parameter $\gamma_{d}$ is a dimension dependent constant that takes values $\gamma_{2}=1 / \pi$ and $\gamma_{3}=1 / 4, \alpha$ is a parameter governing the internal reflection at the boundary $\partial \Omega$, and $\hat{n}$ is an outward normal to the boundary.

A typical TD-DOT measurement setup collects a time-varying boundary exitance $\Gamma(t, r), t=1, \ldots, T$ at detector positions $r$ where $T$ is the temporal range of the output signal. The exitance can be solved from fluence as

$$
\Gamma(r, t)=-\frac{1}{d\left(\mu_{\mathrm{a}}(r)+\mu_{\mathrm{s}}^{\prime}(r)\right)} \frac{\partial \Phi(r, t)}{\partial \hat{n}}=\frac{2 \gamma_{d}}{\alpha} \Phi(r, t) .
$$

In this work, the numerical approximation of the model (1) is based on a finite element method (FEM), following the framework derived in [19, 42]. In the FE-approximation, the domain $\Omega$ is divided into $N_{e}$ non-overlapping elements joined at $N_{n}$ vertex nodes. The photon fluence at a time instance $t$, in a finite dimensional basis is approximated as

$$
\Phi(r, t) \approx \Phi^{\mathrm{h}}(r, t)=\sum_{k=1}^{\mathrm{N}_{\mathrm{n}}} \phi_{k}(t) \psi_{k}(r) \in \Omega^{\mathrm{h}}
$$

where $\Omega^{\mathrm{h}}$ is a finite dimensional subspace spanned by basis functions $\psi_{k}(r), k=1 \ldots \mathrm{N}_{\mathrm{n}}$, and $\phi_{k}(t), k=1 \ldots \mathrm{N}_{\mathrm{n}}$ is the photon fluence at the nodes of the FE-discretisation. We write finite dimensional approximations for $\mu_{\mathrm{a}}(r)$ and $\mu_{\mathrm{s}}^{\prime}(r)$

$$
\begin{aligned}
& \mu_{\mathrm{a}}(r) \approx \mu_{\mathrm{a}}^{\mathrm{h}}(r)=\sum_{l=1}^{\mathrm{N}_{\mathrm{n}}} \mu_{\mathrm{a}, l} \psi_{l}(r) \\
& \mu_{\mathrm{s}}^{\prime}(r) \approx \mu_{\mathrm{s}}^{\prime \mathrm{h}}(r)=\sum_{l=1}^{\mathrm{N}_{\mathrm{n}}} \mu_{\mathrm{s}, l} \psi_{l}(r)
\end{aligned}
$$

where $\mu_{\mathrm{a}, l}, \mu_{\mathrm{s}, l}$ denote the absorption and scattering at the nodes of the FE-discretisation.

The FE-approximation of the time-domain diffusion equation (1) is

$$
\mathbf{K} \boldsymbol{\Phi}(t)+\mathbf{M} \frac{1}{c} \frac{\partial \boldsymbol{\Phi}(t)}{\partial t}=\mathbf{Q}(t)
$$

where fluence $\boldsymbol{\Phi}(t)=\left[\phi_{1}(t), \ldots, \phi_{\mathrm{N}_{\mathrm{n}}}(t)\right]^{\mathrm{T}}$, and matrices $\mathbf{K}$ and $\mathbf{M}$ and vector $\mathbf{Q}$ are

$$
\begin{aligned}
\mathbf{K}_{m k}= & \int_{\Omega} \sum_{l=1}^{\mathrm{N}_{\mathrm{n}}} \frac{1}{d\left(\mu_{\mathrm{a}, l}+\mu_{\mathrm{s}, l}\right)} \psi_{l}(r) \nabla \psi_{m}(r) \cdot \nabla \psi_{k}(r) \mathrm{d} r \\
& +\int_{\Omega} \sum_{l=1}^{\mathrm{N}_{\mathrm{n}}} \mu_{\mathrm{a}, l} \psi_{l}(r) \psi_{m}(r) \psi_{k}(r) \mathrm{d} r \\
& +\frac{2 \gamma_{d}}{\alpha} \int_{\partial \Omega} \psi_{m}(r) \psi_{k}(r) \mathrm{d} r \\
\mathbf{M}_{m k}= & \int_{\Omega} \psi_{m}(r) \psi_{k}(r) \mathrm{d} r \\
\mathbf{Q}_{m}= & \int_{\partial \Omega} Q(r, t) \psi_{m}(r) \mathrm{d} r
\end{aligned}
$$

where $m$ and $k$ are the nodal indices $[19,24]$. In this work, we use a finite-difference method to integrate Eq. (6) for a sequence of time steps $t_{i}=i \Delta t(i=1,2, \ldots)$

$$
\begin{aligned}
& {\left[\theta \mathbf{K}+\frac{1}{\Delta t} \mathbf{M}\right] \mathbf{\Phi}\left(t_{i+1}\right)+\left[(1-\theta) \mathbf{K}-\frac{1}{\Delta t} \mathbf{M}\right] \mathbf{\Phi}\left(t_{i}\right) } \\
= & \theta \mathbf{Q}\left(t_{i+1}\right)+(1-\theta) \mathbf{Q}\left(t_{i}\right),
\end{aligned}
$$

where parameter $\theta$ controls the coupling of the adjacent time steps. Here, we set $\theta=0.5$, which corresponds to the CrankNicholson scheme [42].

\section{B. Temporal moments of TD-DOT}

While it is possible to use the full time-resolved data $\Gamma(r, t)$ sampled at discrete time steps, various integral-transform based data types has been considered earlier [18, 19, 21, 42]. These include $n$-th temporal moment

$$
<t^{n}>(r)=\frac{1}{E(r)} \int_{0}^{\infty} t^{n} \Gamma(r, t) \mathrm{d} t
$$

and $n$-th central moment

$$
c_{n}(r)=\frac{1}{E(r)} \int_{0}^{\infty}(t-<t>(r))^{n} \Gamma(r, t) \mathrm{d} t
$$

where

$$
E(r)=\int_{0}^{\infty} \Gamma(r, t) \mathrm{d} t
$$

is the time-integrated intensity used to normalise the moments. This list could be further extended. A list of the different temporal moments used in earlier TD-DOT studies is presented in Table 1. In this work, we consider the use of the first temporal moment (mean time of flight) $\langle t\rangle(r)$, the second central moment (variance) $c_{2}(r)$ and a combination of both of these moments $\left(<t>(r), c_{2}(r)\right)$, since these are the most widely used moments in previous TD-DOT studies.

The FE-approximation of the mean time of flight and variance of boundary data are given by

$$
\begin{aligned}
<\mathbf{t}> & =\frac{\mathbf{m}_{\mathbf{1}}}{\mathbf{m}_{0}} \\
\mathbf{c}_{2} & =\frac{\mathbf{m}_{\mathbf{2}}}{\mathbf{m}_{0}}-\left(\frac{\mathbf{m}_{\mathbf{1}}}{\mathbf{m}_{0}}\right)^{2}
\end{aligned}
$$

where $\langle\mathbf{t}\rangle=\left[\left\langle t>_{1}, \ldots,\left\langle t>_{N_{m}}\right]^{\mathrm{T}}, \mathbf{c}_{2}=\left[c_{2,1}, \ldots, c_{2, N_{m}}\right]^{\mathrm{T}}\right.\right.$, and $\mathrm{N}_{\mathrm{m}}$ is the number of measurements. Further,

$$
\begin{aligned}
& \mathbf{m}_{\mathbf{0}}=\mathcal{M}\left(\mathbf{K}^{-1} \mathbf{Q}\right) \\
& \mathbf{m}_{\mathbf{1}}=\mathcal{M}\left(\mathbf{K}^{-1}\left(\mathbf{M}\left(\mathbf{K}^{-1} \mathbf{Q}\right)\right)\right) \\
& \mathbf{m}_{\mathbf{2}}=\mathcal{M}\left(2 \mathbf{K}^{-1}\left(\mathbf{M}\left(\mathbf{K}^{-1}\left(\mathbf{M}\left(\mathbf{K}^{-1} \mathbf{Q}\right)\right)\right)\right)\right)
\end{aligned}
$$

as derived in [20]. Here, $\mathbf{K}$ and $\mathbf{M}$ are the FE-matrices in Eqs. (7) and (8), $\mathcal{M}$ is a discretised boundary projection operator in Eq. (2) that maps the FE-solution into measurable data at sensor locations, and $\mathbf{Q}$ is the FE-matrix for the time integrated source $\mathbf{Q} \equiv \int_{0}^{\infty} \mathbf{Q}(t) \mathrm{dt}$.

\section{Truncated Fourier-series approximation}

Truncated Fourier-series approximation of a function $f(t)$ can be expressed as

$$
f(t)=\sum_{k=-N_{\omega}}^{N_{\omega}} f\left(\omega_{k}\right) \exp \left(\mathrm{i} \omega_{k} t\right)
$$


where $\mathrm{i}$ is the imaginary unit, $N_{\omega}$ is the number of Fourier frequencies $\omega_{k}=2 \pi k / \mathcal{T}$ and $f\left(\omega_{k}\right)$ are Fourier coefficients computed with operator $\mathcal{L}(\cdot)$ as

$$
f\left(\omega_{k}\right)=\mathcal{L}(f(t))=\frac{1}{\mathcal{T}} \int_{\mathcal{T}_{a}}^{\mathcal{T}_{b}} f(t) \exp \left(-\mathrm{i} \omega_{k} t\right) \mathrm{d} t,
$$

where $\mathcal{T}_{a}$ and $\mathcal{T}_{b}$ define the interval $\left[\mathcal{T}_{a}, \mathcal{T}_{b}\right]$ where $f(t)$ is approximated and $\mathcal{T}=\mathcal{T}_{b}-\mathcal{T}_{a}$.

The truncated Fourier-series approximation of the DA can be derived similarly as the truncated Fourier-series approximation of the radiative transfer equation in [43]. The incident light $Q(r, t)$ in Eq. (1) is presumed to be real valued and separable into spatial and temporal parts as $Q(r, t)=Q(r) Q(t)$. The temporal shape $Q(t)$ is approximated as a truncated Fourier-series with Fourier coefficients $Q\left(\omega_{k}\right)$. Applying Fourier operator (20) on the time-domain DA (1) with the above assumptions, results in the frequency domain DA

$$
\left\{\begin{array}{l}
\left(-\nabla \cdot \frac{1}{d\left(\mu_{\mathrm{a}}(r)+\mu_{\mathrm{s}}^{\prime}(r)\right)} \nabla+\mu_{\mathrm{a}}+\frac{\mathrm{i} \omega_{k}}{c}\right) \Phi\left(r, \omega_{k}\right)=0, r \in \Omega \\
\Phi\left(r, \omega_{k}\right)+\frac{1}{2 \gamma_{d}} \frac{1}{d\left(\mu_{\mathrm{a}}(r)+\mu_{\mathrm{s}}^{\prime}(r)\right)} \alpha \frac{\partial \Phi\left(r, \omega_{k}\right)}{\partial \hat{n}}=\left\{\begin{array}{l}
\frac{Q(r) Q\left(\omega_{k}\right)}{\gamma_{d}}, r \in \\
0, r \in \partial \Omega \backslash s
\end{array}\right.
\end{array}\right.
$$

Further, the frequency domain exitance can be solved from the fluence as

$$
\Gamma\left(r, \omega_{k}\right)=-\frac{1}{d\left(\mu_{\mathrm{a}}(r)+\mu_{\mathrm{s}}^{\prime}(r)\right)} \frac{\partial \Phi\left(r, \omega_{k}\right)}{\partial \hat{n}}=\frac{2 \gamma_{d}}{\alpha} \Phi\left(r, \omega_{k}\right)
$$

Solving equation (21) for multiple frequencies allows estimation of the time-domain solution of the photon fluence by using the truncated Fourier-series approximation as [24]

$$
\Phi(r, t)=\sum_{k=-N_{\omega}}^{N_{\omega}} \Phi\left(r, \omega_{k}\right) \exp \left(\mathrm{i} \omega_{k} t\right),
$$

where $\Phi\left(r, \omega_{-k}\right)=\Phi\left(r, \omega_{k}\right)^{*}$. Thus, the frequency domain DA needs to be solved for $N_{\omega}+1$ frequency components $\omega_{k}$ with $k=0, \ldots, N_{\omega}$. The truncated Fourier series approximation (19) operates by taking into account the $N_{\omega}+1$ smallest frequencies of the incident light. This approach is proper when the frequency spectrum has the most of the energy at low frequencies.

Utilising the relation between the time-domain and frequency-domain light transport models, it is possible to convert the measured time-resolved data to frequency-domain, and to use that data at one or several frequencies [24]. In that case, light transport can be modelled directly in frequency-domain using (21)-(22). The FE-approximation of the frequency-domain DA (21) is given by

$$
\mathbf{K} \boldsymbol{\Phi}\left(\omega_{k}\right)+\mathbf{M} \frac{\mathrm{i} \omega_{k}}{c} \boldsymbol{\Phi}\left(\omega_{k}\right)=\mathbf{Q}\left(\omega_{k}\right)
$$

where fluence $\boldsymbol{\Phi}\left(\omega_{k}\right)=\left[\phi_{1}\left(\omega_{k}\right), \ldots, \phi_{\mathrm{N}_{\mathrm{n}}}\left(\omega_{k}\right)\right]^{\mathrm{T}}$, and matrices $\mathbf{K}, \mathbf{M}$ and vector $\mathbf{Q}$ are as in (7)-(9). Furthermore, the discretised boundary exitance (22) can be solved using the boundary projection operator $\mathcal{M}$ as

$$
\Gamma\left(\omega_{k}\right)=\mathcal{M} \boldsymbol{\Phi}\left(\omega_{k}\right) .
$$

It should be noted that, for light sources with a finite temporal length, the measurable output $\Gamma(t)$ can be expressed as a convolution $(*)$ of the exitance due to a delta function source $\Gamma_{\delta}(r, t)$ and the source $Q(t)$

$$
\Gamma(t)=\Gamma_{\delta}(t) * Q(t) .
$$

This implies that taking a Fourier transform results in a pointwise product $(\cdot)$ of their Fourier transforms (by convolution theorem) as

$$
\mathcal{L}(\Gamma(t))=\mathcal{L}\left(\Gamma_{\delta}(t)\right) \cdot \mathcal{L}(Q(t)) .
$$

This needs to be taken into account by deconvolution of the source when data is transferred to frequency domain and operated there. In the case of the temporal moments, the effect of the finite temporal length of the light source is taken into account when the moments are scaled with the time-integrated intensity in Eq. (13).

\section{INVERSE PROBLEM}

A discrete observation model for time-domain DOT in the presence of additive noise is

$$
y_{t}=A_{t}\left(\mu_{\mathrm{a}}, \mu_{\mathrm{s}}^{\prime}\right)+e_{t}
$$

where $y_{t}=\left[y_{t, 1}, \ldots, y_{t, N_{\mathrm{m}}}\right]^{\mathrm{T}} \in \mathbb{R}^{\mathcal{T} \mathrm{N}_{\mathrm{m}}}$ is a vector of measured exitances $y_{t, r_{m}} \in \mathbb{R}^{\mathcal{T}}$ at detector positions $r_{m}$ and time instances $t=1, \ldots, \mathcal{T}$. Further, $\mu_{\mathrm{a}}=\left[\mu_{\mathrm{a}, 1}, \ldots, \mu_{\mathrm{a}, \mathrm{N}_{\mathrm{n}}}\right] \in \mathbb{R}^{\mathrm{N}_{\mathrm{n}}}$ and $\mu_{\mathrm{s}}^{\prime}=\left[\mu_{\mathrm{s}, 1}, \ldots, \mu_{\mathrm{s}, \mathrm{N}_{\mathrm{n}}}\right] \in \mathbb{R}^{\mathrm{N}_{\mathrm{n}}}$ are discretised absorption and scattering coefficients, $A_{t}: \mathbb{R}^{2 \mathrm{~N}_{\mathrm{n}}} \mapsto \mathbb{R}^{\mathcal{T} \mathrm{N}_{\mathrm{m}}}$ is the discretised forward operator corresponding to the time-domain DA (1)-(2), and $e_{t} \in \mathbb{R}^{\mathcal{T} \mathrm{N}_{\mathrm{m}}}$ denotes the noise.

Furthermore, we write an observation model for the mean time of light and second central moment as

$$
\begin{aligned}
y_{<t>} & =A_{<t>}\left(\mu_{\mathrm{a}}, \mu_{\mathrm{s}}^{\prime}\right)+e_{<t>} \\
y_{c_{2}} & =A_{\mathcal{c}_{2}}\left(\mu_{\mathrm{a}}, \mu_{\mathrm{s}}^{\prime}\right)+e_{c_{2}}
\end{aligned}
$$

where $y_{<t>}=\left[y_{<t>, 1}, \ldots, y_{<t>, N_{m}}\right]^{\mathrm{T}} \in \mathbb{R}^{\mathrm{N}_{\mathrm{m}}}$ and $y_{c_{2}}=$ $\left[y_{c_{2}, 1}, \ldots, y_{c_{2}, N_{\mathrm{m}}}\right]^{\mathrm{T}} \in \mathbb{R}^{\mathrm{N}_{\mathrm{m}}}$ are vectors of temporal moments of the measured time-resolved data, $A_{<t>}: \mathbb{R}^{2 \mathrm{~N}_{\mathrm{n}}} \mapsto \mathbb{R}^{\mathrm{N}_{\mathrm{m}}}$ and $A_{c_{2}}: \mathbb{R}^{2 \mathrm{~N}_{\mathrm{n}}} \mapsto \mathbb{R}^{\mathrm{N}_{\mathrm{m}}}$ are the discretised forward operators corresponding to the models (11)-(13), and $e_{<t>} \in \mathbb{R}^{\mathrm{N}_{\mathrm{m}}}$ and $e_{\mathcal{C}_{2}} \in \mathbb{R}^{\mathrm{N}_{\mathrm{m}}}$ denote the noise.

In frequency domain, the typical data types considered are the logarithm of amplitude and phase delay

$$
y_{\mathrm{FT}}=\left(\begin{array}{l}
\operatorname{Re} \\
\operatorname{Im}
\end{array}\right) \log y_{\omega_{k}}
$$

where $y_{\mathrm{FT}}=\left[\operatorname{Re} \log y_{\omega_{k}, 1}, \ldots, \operatorname{Re} \log y_{\omega_{k}, \mathrm{~N}_{\mathrm{m}}}, \operatorname{Im} \log y_{\omega_{k}, 1}, \ldots\right.$, Im $\left.\log y_{\omega_{k}, N_{\mathrm{m}}}\right]^{\mathrm{T}} \in \mathbb{R}^{2 \mathrm{~N}_{\mathrm{m}}}$ of frequency domain data $y_{\omega_{k}}$ at one or several frequencies $\omega_{k}$.

In the frequency-domain DOT systems, the frequency domain data at one modulation frequency is measured directly. In the time-resolved systems, the frequency-domain data at one or multiple Fourier frequencies is obtained through Fourier transform and deconvolution of the source pulse

$$
y_{\omega_{k}}=\frac{\mathcal{L}(y(t))}{\mathcal{L}(Q(t))}=\frac{\mathcal{L}(y(t))}{Q_{\omega_{k}}}
$$

where $Q_{\omega_{k}}$ is the Fourier transform of the source pulse as in Eq. (27) [24]. The observation model for the logarithm of amplitude and phase delay are

$$
y_{\mathrm{FT}}=\left(\begin{array}{c}
\operatorname{Re} \\
\operatorname{Im}
\end{array}\right) \log A_{\omega_{k}}\left(\mu_{\mathrm{a}}, \mu_{\mathrm{s}}^{\prime}\right)+e_{\mathrm{FT}}
$$


where $A_{\omega_{k}}: \mathbb{R}^{2 \mathrm{~N}_{\mathrm{n}}} \mapsto \mathbb{C}^{\mathrm{N}_{\mathrm{m}}}$ is the discretised forward operator corresponding to the frequency domain DA (21)-(22) and $e_{\mathrm{FT}} \in$ $\mathbb{R}^{2 \mathrm{~N}_{\mathrm{m}}}$ denotes the noise.

\section{A. Bayesian approach to the inverse problem}

In the Bayesian approach to inverse problems, all the parameters are considered random variables and the uncertainties of their values are encoded into probability density models [44]. Let us consider the observation model of the TD-DOT (28). The solution of the inverse problem is the posterior probability density which is obtained through Bayes' theorem and can be written as

$$
\pi\left(\mu_{\mathrm{a}}, \mu_{\mathrm{s}}^{\prime} \mid y_{t}\right) \propto \pi\left(y_{t} \mid \mu_{\mathrm{a}}, \mu_{\mathrm{s}}^{\prime}\right) \pi\left(\mu_{\mathrm{a}}, \mu_{\mathrm{s}}^{\prime}\right)
$$

where $\pi\left(y_{t} \mid \mu_{\mathrm{a}}, \mu_{\mathrm{s}}^{\prime}\right)$ is the likelihood density and $\pi\left(\mu_{\mathrm{a}}, \mu_{\mathrm{s}}^{\prime}\right)$ is the prior density.

Since we aim at computationally efficient solutions, we compute point estimate(s) from the posterior density, the most typical choice being the maximum a posteriori (MAP) estimate. Assuming that the unknowns $\mu_{\mathrm{a}}$ and $\mu_{\mathrm{s}}^{\prime}$ and noise $e_{t}$ are mutually independent and Gaussian distributed, i.e

$$
\mu_{\mathrm{a}} \sim \mathcal{N}\left(\eta_{\mu_{\mathrm{a}}}, \Gamma_{\mu_{\mathrm{a}}}\right), \quad \mu_{\mathrm{s}}^{\prime} \sim \mathcal{N}\left(\eta_{\mu_{\mathrm{s}}^{\prime}}, \Gamma_{\mu_{\mathrm{s}}^{\prime}}\right), \quad e_{t} \sim \mathcal{N}\left(\eta_{e_{t}}, \Gamma_{e_{t}}\right)
$$

where $\eta_{\mu_{\mathrm{a}}}, \eta_{\mu_{\mathrm{s}}^{\prime}}$ and $\eta_{e_{t}}$ are the means, and $\Gamma_{\mu_{\mathrm{a}}}, \Gamma_{\mu_{\mathrm{s}}^{\prime}}$ and $\Gamma_{e_{t}}$ are the covariance matrices, the MAP estimate is obtained as

$$
\begin{aligned}
\left(\hat{\mu}_{\mathrm{a}}, \hat{\mu}_{\mathrm{s}}^{\prime}\right)= & \underset{\mu_{\mathrm{a}}, \mu_{\mathrm{s}}^{\prime}}{\arg \min }\left\{\frac{1}{2}\left\|L_{e_{t}}\left(y_{t}-A_{t}\left(\mu_{\mathrm{a}}, \mu_{\mathrm{s}}^{\prime}\right)-\eta_{e_{t}}\right)\right\|^{2}\right. \\
& \left.+\frac{1}{2}\left\|L_{\mu_{\mathrm{a}}}\left(\mu_{\mathrm{a}}-\eta_{\mu_{\mathrm{a}}}\right)\right\|^{2}+\frac{1}{2}\left\|L_{\mu_{\mathrm{s}}^{\prime}}\left(\mu_{\mathrm{s}}^{\prime}-\eta_{\mu_{\mathrm{s}}^{\prime}}\right)\right\|^{2}\right\}
\end{aligned}
$$

where $L_{\mu_{\mathrm{a}}}^{\mathrm{T}} L_{\mu_{\mathrm{a}}}=\Gamma_{\mu_{\mathrm{a}}}^{-1}, L_{\mu_{\mathrm{s}}^{\prime}}^{\mathrm{T}} L_{\mu_{\mathrm{s}}^{\prime}}=\Gamma_{\mu_{\mathrm{s}}^{\prime}}^{-1}$ and $L_{e_{t}}^{\mathrm{T}} L_{e_{t}}=\Gamma_{e_{t}}^{-1}$ are matrix square roots such as the Cholesky decomposition.

In this work the prior model for the unknown parameters was chosen to be based on Ornstein-Uhlenbeck process which belongs to the Matérn class of covariance functions [45]. OrnsteinUhlenbeck prior is a Gaussian distribution with the covariance matrix $\Gamma$ defined as

$$
\Gamma_{x, m k}=\sigma_{x}^{2} \exp \left(-\frac{\left\|r_{m}-r_{k}\right\|}{\ell}\right)
$$

where $x$ denotes the unknown parameters (absorption and scattering), $\sigma_{x}$ is the standard deviation, $r_{m}$ and $r_{k}$ are the coordinates of the discretisation points (in this work the locations of the FE-discretisation nodes $m$ and $k$ ), and $\ell$ is the characteristic length scale which controls the spatial range of correlation. It supports correlation between neighborhood discretization points, promoting distributions that can be locally close to homogeneous.

The MAP estimates for the temporal moments and frequency domain data can be derived similarly as for the time-domain data using observation models (29), (30) and (33). They are obtained as

$$
\begin{aligned}
\left(\hat{\mu}_{\mathrm{a}}, \hat{\mu}_{\mathrm{s}}^{\prime}\right)= & \underset{\mu_{\mathrm{a}}, \mu_{\mathrm{s}}^{\prime}}{\arg \min }\left\{\frac{1}{2}\left\|L_{e_{*}}\left(y_{*}-A_{*}\left(\mu_{\mathrm{a}}, \mu_{\mathrm{s}}^{\prime}\right)-\eta_{e_{*}}\right)\right\|^{2}\right. \\
& \left.+\frac{1}{2}\left\|L_{\mu_{\mathrm{a}}}\left(\mu_{\mathrm{a}}-\eta_{\mu_{\mathrm{a}}}\right)\right\|^{2}+\frac{1}{2}\left\|L_{\mu_{\mathrm{s}}^{\prime}}\left(\mu_{\mathrm{s}}^{\prime}-\eta_{\mu_{\mathrm{s}}^{\prime}}\right)\right\|^{2}\right\}
\end{aligned}
$$

where $y_{*}$ denotes the different temporal moments or the frequency domain data $\left(y_{<t>}, y_{c_{2}}, y_{\mathrm{FT}}\right.$ or their combinations), and $A_{*}$ denotes the corresponding discretised forward operators $\left(A_{<t>}, A_{\mathcal{C}_{2}}\right.$ and $\left.(\operatorname{Re} ; \operatorname{Im}) \log A_{\omega_{k}}\right)$. Further, $\eta_{e_{*}}$ and $L_{e_{*}}$ are means and Cholesky decompositions of the Gaussian distributed noise $e_{*} \sim \mathcal{N}\left(\eta_{e_{*}}, \Gamma_{e_{*}}\right)$.

In this work, the minimisation problem (37) using different data types and their combinations is solved using the GaussNewton method utilising a line-search algorithm with a positivity constrain for determining the step length. Calculation of the Jacobians of the different data types for the Gauss-Newton algorithm is described in Appendix A.

\section{B. Difference imaging}

Consider data $y_{*, 1}$ and $y_{*, 2}$ of two DOT measurements obtained from a target with optical parameters $\left(\mu_{\mathrm{a}, 1}, \mu_{\mathrm{s}, 1}^{\prime}\right)$ and $\left(\mu_{\mathrm{a}, 2}, \mu_{\mathrm{s}, 2}^{\prime}\right)$, respectively. The aim in difference imaging is to reconstruct the change in the optical parameters $\left(\delta \mu_{\mathrm{a}}, \delta \mu_{\mathrm{s}}^{\prime}\right)=\left(\mu_{\mathrm{a}, 2}-\mu_{\mathrm{a}, 1}, \mu_{\mathrm{s}, 2}^{\prime}-\right.$ $\left.\mu_{\mathrm{s}, 1}^{\prime}\right)$ based on the difference of the measurements as

$$
y_{*, 2}-y_{*, 1}=J_{*}\left(\mu_{\mathrm{a}, 1}, \mu_{\mathrm{s}, 1}^{\prime}\right)\left(\delta \mu_{\mathrm{a}}, \delta \mu_{\mathrm{s}}^{\prime}\right)+\delta e_{*}
$$

where the Jacobian $J_{*}\left(\mu_{\mathrm{a}, 1}, \mu_{\mathrm{s}, 1}^{\prime}\right)$ is the discrete representation of the Fréchet derivative of the nonlinear mapping $A_{*}\left(\mu_{\mathrm{a}}, \mu_{\mathrm{s}}^{\prime}\right)$. Considering Gaussian distributed optical parameters and noise

$\delta \mu_{\mathrm{a}} \sim \mathcal{N}\left(0, \Gamma_{\delta \mu_{\mathrm{a}}}\right), \quad \delta \mu_{\mathrm{s}}^{\prime} \sim \mathcal{N}\left(0, \Gamma_{\delta \mu_{\mathrm{s}}^{\prime}}\right), \quad \delta e_{*} \sim \mathcal{N}\left(0, \Gamma_{e_{*, 1}}+\Gamma_{e_{*, 2}}\right)$, the MAP estimate for difference imaging using the linear approximation (38) is

$$
\begin{aligned}
\left(\delta \hat{\mu}_{\mathrm{a}}, \delta \hat{\mu}_{\mathrm{s}}^{\prime}\right)=( & \left(J_{*}\left(\mu_{\mathrm{a}, 1}, \mu_{\mathrm{s}, 1}^{\prime}\right)^{\mathrm{T}} \Gamma_{\delta e_{*}}^{-1} J_{*}\left(\mu_{\mathrm{a}, 1}, \mu_{\mathrm{s}, 1}^{\prime}\right)+\Gamma_{\mu_{\mathrm{a}}}^{-1}+\Gamma_{\mu_{\mathrm{s}}^{\prime}}^{-1}\right)^{-1} \\
& \cdot\left(J_{*}\left(\mu_{\mathrm{a}, 1}, \mu_{\mathrm{s}, 1}^{\prime}\right)^{\mathrm{T}} \Gamma_{\delta e_{*}}^{-1}\left(y_{*, 2}-y_{*, 1}\right)\right) .
\end{aligned}
$$

\section{SIMULATIONS}

Simulations were carried out in a Fujitsu Celcius W550 desktop workstation, with Intel®Xeon(R) W-2125 CPU @ 4.00GHz $\times 8$, using MATLAB (R2017b, Mathworks, Natick, MA). The Toast++ software [46] was utilised in the FE-solution of the diffusion equation.

In the numerical studies, the domain $\Omega \subset \mathbb{R}^{2}$ was a circle with a radius of $25 \mathrm{~mm}$. The setup consisted of 16 sources and 16 detectors. The source and detector optodes were modelled as Gaussian surface patches with $2 \mathrm{~mm}$ width, located at equispaced angular intervals on the boundary $\partial \Omega$.

\section{A. Single perturbation study}

To visualize the reconstruction problem utilising the different data types, we studied residuals, i.e. differences between simulated data sets, in a case of a single perturbation inclusion embedded in the $2 \mathrm{D}$ simulation domain. The residual $R$ was defined as

$$
\mathrm{R}=\left\|y_{i}\left(\bar{\mu}_{\mathrm{a}}, \bar{\mu}_{\mathrm{s}}^{\prime}\right)-y_{i}\left(\mu_{\mathrm{a}, 0}, \mu_{\mathrm{s}, 0}^{\prime}\right)\right\|
$$

where $y_{i}$ denotes different temporal moments or frequency domain data $\left(y_{<t>}, y_{c_{2}}, y_{\mathrm{FT}}\right)$. Parameters of the 'reference' data had background optical parameters $\mu_{\mathrm{a}, 0}=0.01 \mathrm{~mm}^{-1}$, $\mu_{\mathrm{s}, 0}^{\prime}=1 \mathrm{~mm}^{-1}$, and one circular inclusion with parameters $\mu_{\mathrm{a}, 0}=0.02 \mathrm{~mm}^{-1}, \mu_{\mathrm{s}, 0}^{\prime}=2 \mathrm{~mm}^{-1}$. Further, multiple data sets were simulated by varying parameters $\bar{\mu}_{\mathrm{a}}$ and $\bar{\mu}_{\mathrm{s}}^{\prime}$ such that the background parameters were the same as for the 'reference' data i.e. $\mu_{\mathrm{a}}=0.01 \mathrm{~mm}^{-1}$ and $\mu_{\mathrm{s}}^{\prime}=1 \mathrm{~mm}^{-1}$, and the inclusion presented multiple absorption and scattering values in the range $\mu_{\mathrm{a}} \in[0.0050 .02] \mathrm{mm}^{-1}$ and $\mu_{\mathrm{s}}^{\prime} \in[0.52] \mathrm{mm}^{-1}$. 

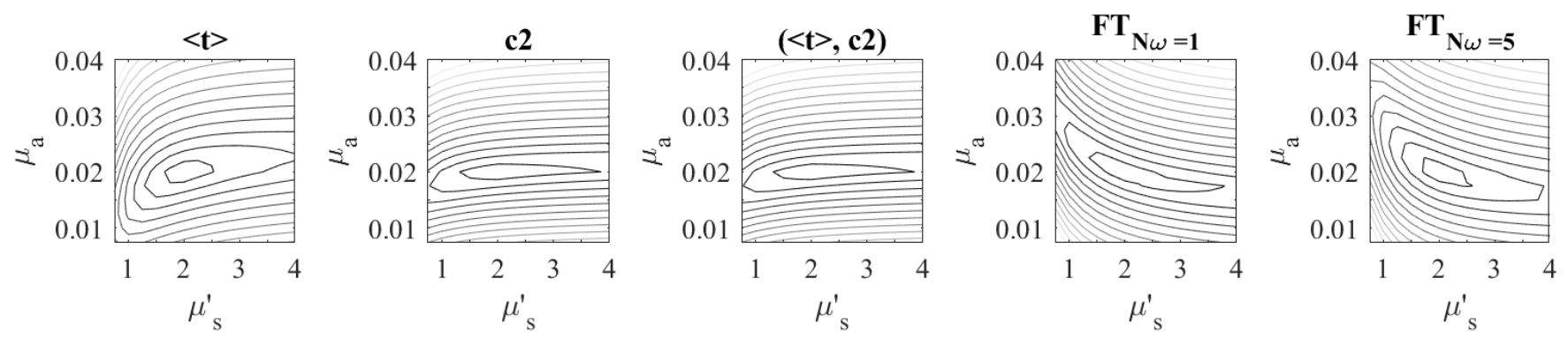

Fig. 1. Contour plots of residuals $R$, Eq. (40), for different data types due to a perturbation in the absorption $\mu_{\mathrm{a}}\left(\mathrm{mm}^{-1}\right)$ and scattering $\mu_{\mathrm{s}}^{\prime}\left(\mathrm{mm}^{-1}\right)$ parameters. The studied data types were the mean time of flight $\langle t\rangle$, variance $c_{2}$, their combination $\left(\langle t\rangle, c_{2}\right)$, and Fourier transformed data at one $\mathrm{FT}_{\mathrm{N} \omega=1}$ and five $\mathrm{FT}_{\mathrm{N} \omega=5}$ frequencies.

Contour plots of the residuals are showed in Fig. 1. Similar plots of $y<t>, y_{c_{2}}$ and some other data types have also been studied earlier in Ref. [21]. As it can be seen, all data types show an elongated 'valley' of low data error. Note that the orientation and steepness of the valley differs for different data types, which affects the relative sensitivity to changes in absorption and scattering. The residual of $\langle t\rangle$ is almost equivalent for $\mu_{\mathrm{a}}$ and $\mu_{\mathrm{s}}^{\prime}$. However, the residuals of $c_{2}$ and $\left(\langle t\rangle, c_{2}\right)$ are more sensitive to variations in $\mu_{\mathrm{a}}$. Frequency domain data with five frequencies shows the most clearly defined minimum, indicating that it may provide the best means to separate $\mu_{\mathrm{a}}$ and $\mu_{\mathrm{s}}^{\prime}$.

\section{B. Data generation}

We studied four different target distributions. These are shown on the first row of Fig. 2. These parameters were used to simulate the data sets that were used both in absolute imaging and in difference imaging studies. For difference imaging, the reference data was simulated using homogeneous optical properties $\mu_{\mathrm{a}}=$ $0.01 \mathrm{~mm}^{-1}$ and $\mu_{\mathrm{s}}^{\prime}=1 \mathrm{~mm}^{-1}$ that correspond to the background optical parameters of the first two numerical phantoms. The difference distributions are shown on the first row of Fig. 4.

For all targets, the time-resolved data was simulated using FE-approximation of the DA (1) in a mesh with 1369 nodes and 2622 triangular elements. The temporal discretisation was 1 ps, the source pulses had 10 ps duration, and the temporal range was specified as 10000 ps. The total number of the simulated time-resolved measurements was 12800000 (256 combination of sources and detectors, 50000 time steps). The temporal moments and Fourier transformed data were computed from these timeresolved measurements. Random measurement noise $e$, that was drawn from a zero-mean Gaussian distribution

$$
\pi(e)=\mathcal{N}\left(0, \Gamma_{e}\right), \quad \Gamma_{e}=\operatorname{diag}\left(\sigma_{e, 1}^{2}, \ldots, \sigma_{e, \mathrm{~N}_{\mathrm{m}}}^{2}\right)
$$

where the standard deviations $\sigma_{e, 1}, \ldots, \sigma_{e, \mathrm{~N}_{\mathrm{m}}}$ were specified as $1 \%$ of the simulated noise free transformed data, was added to the transformed data.

To study the solution of the inverse problem with different data types systematically, we generated a set of randomly simulated targets. For this, 100 samples of optical parameter distributions were drawn from the Ornstain-Uhlenback prior model where the mean of the prior was set as $\eta_{\mu_{\mathrm{a}}}=0.01 \mathrm{~mm}^{-1}$ and $\eta_{\mu_{\mathrm{s}}^{\prime}}=1 \mathrm{~mm}^{-1}$, the standard deviation was set as $\sigma_{\mu_{\mathrm{a}}}=$ $0.001 / 3 \mathrm{~mm}^{-1}$ and $\sigma_{\mu_{\mathrm{s}}^{\prime}}=1 / 3 \mathrm{~mm}^{-1}$, and the characteristic length was $\ell=8 \mathrm{~mm}$. Examples of targets drawn from the prior are shown on the first row, last two columns of Figs. 2 and
4. These simulated optical parameter distributions were used to simulate samples of time-resolved data, and further generate samples of different data types.

\section{Image reconstruction}

In the computation of the MAP estimates for absolute imaging (37) and difference imaging (39), using the temporal moments and the Fourier transformed data, a FE-mesh with 1123 nodes and 2142 elements was used.

The measurement noise mean and covariance were assumed known for each data type. The parameters of OrnsteinUhlenbeck prior were chosen such that, for absolute imaging, the prior means $\left(\eta_{\mu_{\mathrm{a}}}, \eta_{\mu_{\mathrm{s}}^{\prime}}\right)$ were set as the background optical parameters and the standard deviations $\left(\sigma_{\mu_{\mathrm{a}}}, \sigma_{\mu_{\mathrm{s}}^{\prime}}\right)$ were set such that maximum target values corresponded to three standard deviations from the background. For difference imaging, the prior means $\left(\eta_{\delta \mu_{\mathrm{a}}}, \eta_{\delta \mu_{\mathrm{s}}^{\prime}}\right)$ were set to zero and the standard deviations $\left(\delta \sigma_{\mu_{\mathrm{a}}}, \delta \sigma_{\mu_{\mathrm{s}}^{\prime}}\right)$ were set such that maximum target values corresponded to five standard deviations from the background. The characteristic length scale was set as $\ell=8 \mathrm{~mm}$, corresponding approximately to the size of the inhomogeneities in the targets used.

In absolute imaging, the initial value for the Gauss-Newton algorithm was chosen to be the mean of the prior. The iteration was considered converged, when the change in the minimised functional was smaller than $10^{-12}$ in two consecutive iterations.

The accuracy of the estimates were evaluated by computing relative errors

$$
\begin{aligned}
\mathrm{E}_{\mu_{\mathrm{a}}} & =100 \% \cdot \frac{\left\|\hat{\mu}_{\mathrm{a}}-\mu_{\mathrm{a}}^{\text {target }}\right\|}{\left\|\mu_{\mathrm{a}}{ }^{\text {target }}\right\|} \\
\mathrm{E}_{\mu_{\mathrm{s}}^{\prime}} & =100 \% \cdot \frac{\left\|\hat{\mu}_{\mathrm{s}}^{\prime}-\mu_{\mathrm{s}}^{\text {target }}\right\|}{\left\|\mu_{\mathrm{s}}^{\prime \text { target }}\right\|} \\
\mathrm{E}_{\delta \mu_{\mathrm{a}}} & =100 \% \cdot \frac{\left\|\delta \hat{\mu}_{\mathrm{a}}-\delta \mu_{\mathrm{a}}^{\text {target }}\right\|}{\left\|\delta \mu_{\mathrm{a}}^{\text {target }}\right\|} \\
\mathrm{E}_{\delta \mu_{\mathrm{s}}^{\prime}} & =100 \% \cdot \frac{\left\|\delta \hat{\mu}_{\mathrm{s}}^{\prime}-\delta \mu_{\mathrm{s}}^{\prime \text { target }}\right\|}{\left\|\delta \mu_{\mathrm{s}}^{\prime \text { target }}\right\|}
\end{aligned}
$$

where $\mu_{\mathrm{a}}{ }^{\text {target }}, \mu_{\mathrm{s}}^{\prime \text { target }}, \delta \mu_{\mathrm{a}}{ }^{\text {target }}$ and $\delta \mu_{\mathrm{s}}^{\prime \text { target }}$ are the simulated target distributions for absorption and scattering, and $\hat{\mu}_{\mathrm{a}}, \hat{\mu}_{\mathrm{s}}^{\prime}$, $\delta \hat{\mu}_{\mathrm{a}}$ and $\delta \hat{\mu}_{\mathrm{s}}^{\prime}$ are the estimated parameters interpolated to the simulation grid. 
Target 1

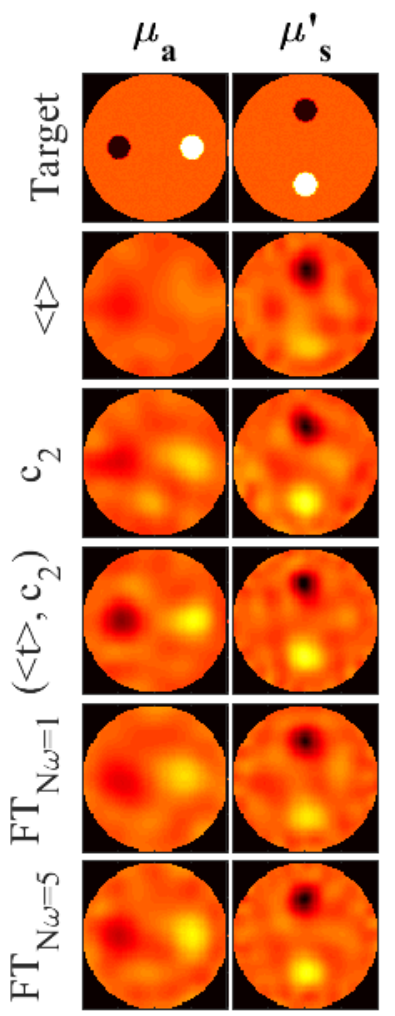

Target 2
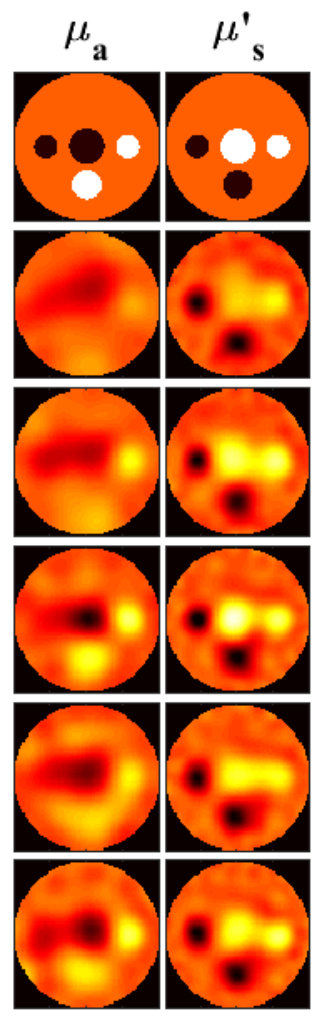

$\mu_{\mathrm{a}}=0$

$\mu_{\mathrm{s}}^{\prime}=0$
Target 3
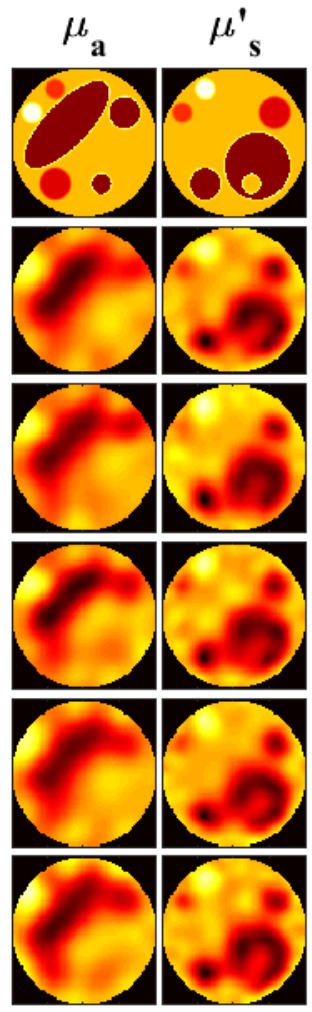

$\mu_{\mathrm{a}}=0.02$

$\mu^{\prime}{ }_{\mathrm{s}}=2$
Target 4
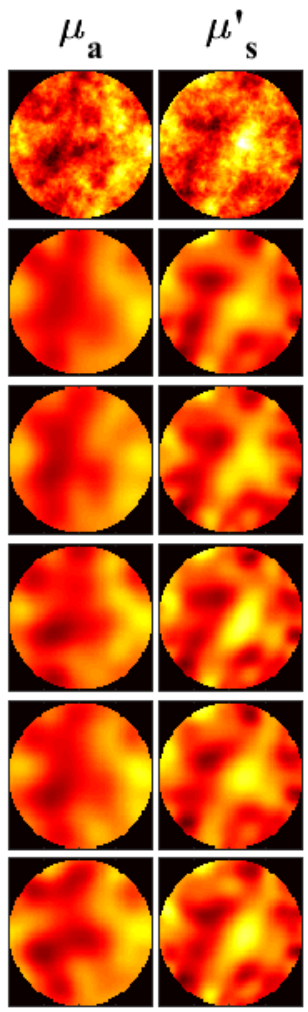

Fig. 2. Absorption $\mu_{\mathrm{a}}\left(\mathrm{mm}^{-1}\right)$ and scattering $\mu_{\mathrm{s}}^{\prime}\left(\mathrm{mm}^{-1}\right)$ distributions. First row: target distribution. From second to sixth rows: estimated parameters obtained using data types mean time of flight $<t>$, variance $c_{2}$, their combination $\left(<t>, c_{2}\right)$, and Fourier transformed data at one $\mathrm{FT}_{\mathrm{N} \omega=1}$ and five $\mathrm{FT}_{\mathrm{N} \omega=5}$ frequencies.

Table 2. Relative errors $E_{\mu_{\mathrm{a}}}(\%)$ and $E_{\mu_{\mathrm{s}}^{\prime}}(\%)$ of the absorption and scattering estimates shown in Fig. 2. The utilised data types were the mean time of flight $\langle t\rangle$, variance $c_{2}$, their combination $\left(\langle t\rangle, c_{2}\right)$, and Fourier transformed data at one $\mathbf{F T}_{\mathrm{N}_{\omega}=1}$ and five $\mathbf{F T}_{\mathrm{N}_{\omega}=5}$ frequencies.

\begin{tabular}{lcccccccc}
\hline & \multicolumn{2}{c}{ Target 1 } & \multicolumn{2}{c}{ Target 2 } & \multicolumn{2}{c}{ Target 3 } & \multicolumn{2}{c}{ Target 4 } \\
\cline { 2 - 8 } Type & $E_{\mu_{\mathrm{a}}}$ & $E_{\mu_{\mathrm{s}}^{\prime}}$ & $E_{\mu_{\mathrm{a}}}$ & $E_{\mu_{\mathrm{s}}^{\prime}}$ & $E_{\mu_{\mathrm{a}}}$ & $E_{\mu_{\mathrm{s}}^{\prime}}$ & $E_{\mu_{\mathrm{a}}}$ & $E_{\mu_{\mathrm{s}}^{\prime}}$ \\
\hline$<t>$ & 16 & 13 & 29 & 20 & 23 & 17 & 18 & 12 \\
$c_{2}$ & 15 & 14 & 24 & 17 & 22 & 18 & 17 & 13 \\
$<t>, c_{2}$ & 14 & 12 & 19 & 15 & 20 & 16 & 16 & 11 \\
$\mathrm{FT}_{\mathrm{N}_{\omega}=1}$ & 15 & 12 & 22 & 18 & 21 & 16 & 17 & 12 \\
$\mathrm{FT}_{\mathrm{N}_{\omega}=5}$ & 14 & 11 & 20 & 17 & 19 & 15 & 16 & 11 \\
\hline & & & & & & & &
\end{tabular}

\section{RESULTS}

\section{A. Absolute imaging}

Obtained MAP estimates for the four simulation targets utilising temporal moments and Fourier transformed data, are shown in Fig. 2. The corresponding relative errors of the estimates are listed in Table 2.
As it can be seen, the estimates obtained using a single temporal moment as data show spatial blurring and lower contrast when compared to the target optical properties. This is especially evident for the absorption estimates. Variance data gives a slightly better contrast than the mean time of flight.

Utilising both mean time of flight and variance data provides better contrast and localisation than usage of individual moments. Further, the relative errors of the absorption and scattering estimates are smaller than those obtained using single temporal moments.

When compared to utilising Fourier transformed data (33), usage of one Fourier frequency provides similar quality reconstruction as usage of two temporal moments. The relative errors obtained using the mean time of light and variance data are smaller than using the Fourier transformed data at one frequency for all other targets except scattering estimates of target 1 and target 3 that give equal size relative errors.

Using five Fourier frequencies improves all reconstructions. For example, the highly scattering inclusions in target 2 can now be distinguished as two separate inclusions. Furthermore, the relative errors are smaller than using any other data types for all other targets except for the target 2 where the combined mean time of flight and variance data gives the lowest relative errors.

Statistics of the relative errors computed from the MAP estimates from 100 targets drawn from the prior distribution are shown in Fig. 3. As it can be seen, using a single data type 


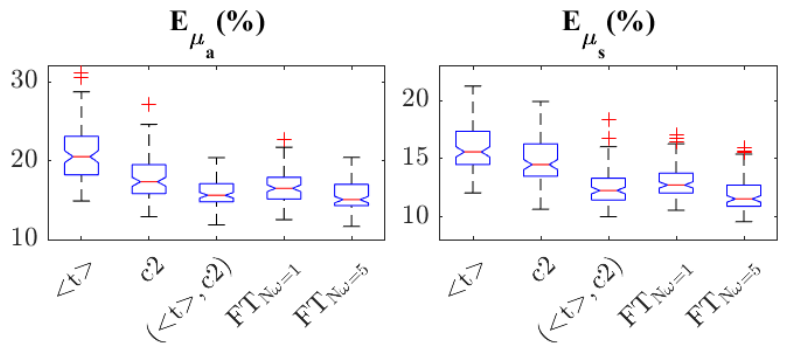

Fig. 3. Statistics of the relative errors $E_{\mu_{\mathrm{a}}}(\%)$ and $E_{\mu_{\mathrm{s}}^{\prime}}(\%)$ of the absorption and scattering estimates from 100 target samples. The utilised data types were the mean time of flight $\langle t\rangle$, variance $c_{2}$, their combination $\left(\langle t\rangle, c_{2}\right)$, and Fourier transformed data at one $\mathrm{FT}_{\mathrm{N}_{\omega}=1}$ and five $\mathrm{FT}_{\mathrm{N}_{\omega}=5}$ frequencies.

produces worse absorption and scattering estimates than using a combination of multiple data types. Furthermore, combination of the mean time of flight and variance as data give a better estimation accuracy for absorption than utilising a single Fourier frequency. For scattering, on the other hand, the frequency domain data performs better than the combined mean time of flight and variance. The best estimation accuracy is obtained using frequency domain data at five Fourier frequencies.

\section{B. Difference imaging}

Difference imaging estimates for the simulated four targets utilising the temporal moments and Fourier transformed data are shown in Fig. 4. The corresponding relative errors of the estimates are listed in Table 3.

As it can be seen, the difference imaging estimates using single temporal moments show spatial blurring and low contrast compared to the target optical properties, similarly as in absolute imaging. Using the variance as data gives slightly better contrast and localisation of the absorption estimates than the mean time of flight. Utilising both mean time of flight and standard deviation, provides a better contrast and location than the individual moments, which is consistent with previous studies in Refs. [16, 21].

Utilising Fourier transformed data at one frequency provides lower estimation accuracy than utilising both mean time of flight and variance for target 1 , for both absorption and scattering. However, in targets 3 and 4, the estimation accuracy for both parameters is higher when using the Fourier transformed data. Furthermore, utilising the Fourier transformed data at five frequencies provides better or as good absorption and scattering estimates as utilising data at one frequency for all targets except in the case of scattering estimates of target 2 .

Statistics of relative errors computed from the difference estimates of 100 targets drawn from the prior distribution are shown in Fig. 5. As it can be seen, using multiple data types provides better estimates than using the mean time of flight or variance data alone. Furthermore, utilising Fourier transformed data at one frequency provides smaller relative errors than using both mean time of flight and variance. Utilising five Fourier frequencies, improves the estimates even more, giving the lowest relative errors.

\section{DISCUSSION AND CONCLUSIONS}

In this work, we studied utilising temporal moments mean time of flight and variance and Fourier transformed data using one and five frequencies in time-resolved DOT. The approaches were
Table 3. Relative errors $E_{\delta \mu_{\mathrm{a}}}(\%)$ and $E_{\delta \mu_{\mathrm{s}}^{\prime}}(\%)$ of the absorption and scattering difference estimates shown in Fig. 4. The utilised data types were the mean time of flight $\langle t\rangle$, variance $c_{2}$, their combination $\left(\langle t\rangle, c_{2}\right)$, and Fourier transformed data at one $\mathbf{F T}_{\mathrm{N}_{\omega}=1}$ and five $\mathbf{F T}_{\mathrm{N}_{\omega}=5}$ frequencies.

\begin{tabular}{lcccccccc}
\hline & \multicolumn{2}{c}{ Target 1 } & \multicolumn{2}{c}{ Target 2 } & \multicolumn{2}{c}{ Target 3 } & \multicolumn{2}{c}{ Target 4 } \\
\cline { 2 - 8 } Type & $E_{\delta \mu_{\mathrm{a}}}$ & $E_{\delta \mu_{\mathrm{s}}^{\prime}}$ & $E_{\delta \mu_{\mathrm{a}}}$ & $E_{\delta \mu_{\mathrm{s}}^{\prime}}$ & $E_{\delta \mu_{\mathrm{a}}}$ & $E_{\delta \mu_{\mathrm{s}}^{\prime}}$ & $E_{\delta \mu_{\mathrm{a}}}$ & $E_{\delta \mu_{\mathrm{s}}^{\prime}}$ \\
\hline$<t>$ & 89 & 85 & 101 & 81 & 86 & 68 & 78 & 56 \\
$c_{2}$ & 80 & 78 & 91 & 96 & 67 & 141 & 76 & 67 \\
$<t>, c_{2}$ & 75 & 79 & 79 & 91 & 81 & 72 & 70 & 61 \\
$\mathrm{FT}_{\mathrm{N}_{\omega}=1}$ & 82 & 83 & 83 & 81 & 69 & 60 & 63 & 57 \\
$\mathrm{FT}_{\mathrm{N}_{\omega}=5}$ & 74 & 78 & 73 & 83 & 68 & 58 & 63 & 53 \\
\hline
\end{tabular}

tested with several target optical parameter distributions, both for absolute and difference imaging. In addition to different reconstructions and their relative errors, a trial with 100 samples was performed to study the estimates obtained using different data types systematically.

The simulations show that utilising two temporal moments and Fourier transformed data at one frequency give almost similar quality reconstructions with relative errors on a similar level. For targets studied in the work, the absolute images with temporal moments are slightly better than with frequency domain data. On the other hand, in difference imaging, Fourier transformed data provides better estimates than the temporal moments. Both in absolute and difference imaging, utilising Fourier transformed data at five frequencies provides the best estimates with good image quality and contrast, and most accurate estimates. This is in line with our previous study [24], where the accuracy of the estimates increased when the number of Fourier frequencies was increased, but they did not change substantially after usage of three or four Fourier frequencies.

Further comparing to the previous work in [24], an alternative to using the logarithm of amplitude and phase delay (31) as data types for Fourier transformed data, the real and imaginary parts of the frquency domain data $y_{\omega_{k}}$ could be used

$$
y_{\mathrm{RI}}=\left(\begin{array}{l}
\operatorname{Re} \\
\operatorname{Im}
\end{array}\right) y_{\omega_{k}}
$$

where $y_{\mathrm{RI}}=\left[\operatorname{Re} y_{\omega_{k}, 1}, \ldots, \operatorname{Re} y_{\omega_{k}, \mathrm{~N}_{\mathrm{m}}}, \operatorname{Im} y_{\omega_{k}, 1}, \ldots, \operatorname{Im} y_{\omega_{k}, \mathrm{~N}_{\mathrm{m}}}\right]^{\mathrm{T}} \in$ $\mathbb{R}^{2 \mathrm{~N}_{\mathrm{m}}}$. However, we demonstrate in Appendix $\mathrm{B}$ that this can cause image artefacts in situations where large phase delays are present, due to the periodic nature of the data with respect to the phase.

The computation times of the MAP estimates of the target 1 are shown in Table 4. These show that the computation of the estimates using frequency domain data types are significantly lower than utilising the temporal moments. As shown in Appendix $\mathrm{A}$, calculation of the Jacobian matrices of the temporal moments $\langle t\rangle$ and $c_{2}$ is computationally complex, requiring several large matrix operations. Hence, compared to the temporal moments, the frequency domain computations are inexpensive and their implementation is relatively straightforward. This can be useful in practical situations, for example online imaging of optical parameters using TD-DOT. 
Target 1

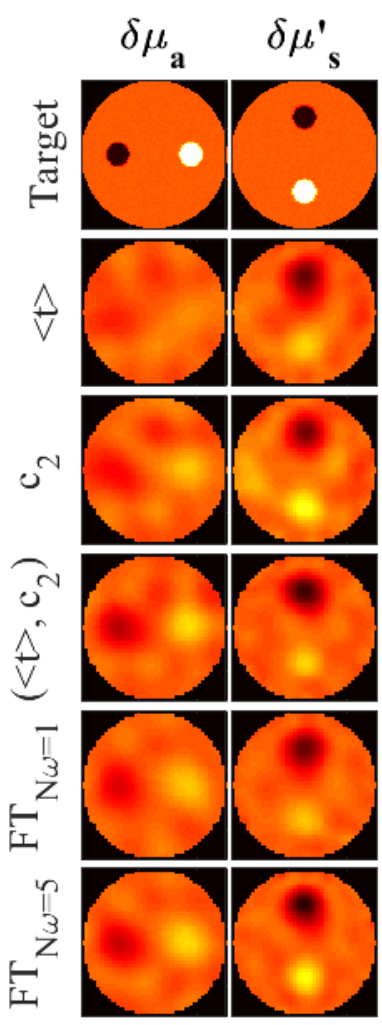

Target 2
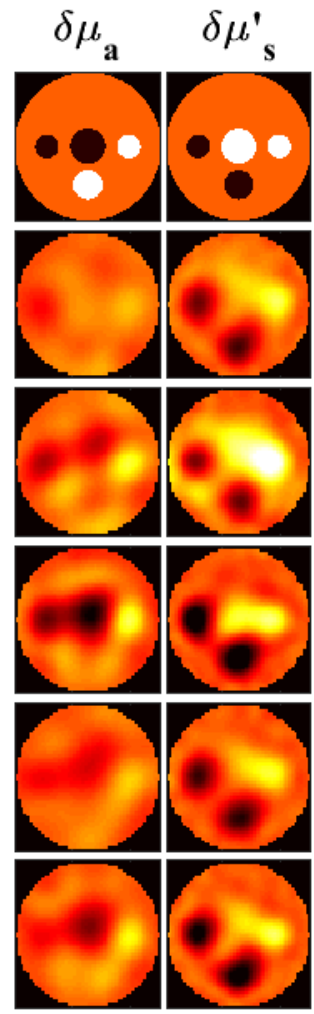

$\mu_{\mathrm{a}}=-0.01$ $\mu_{\mathrm{s}}^{\prime}=-1$
Target 3
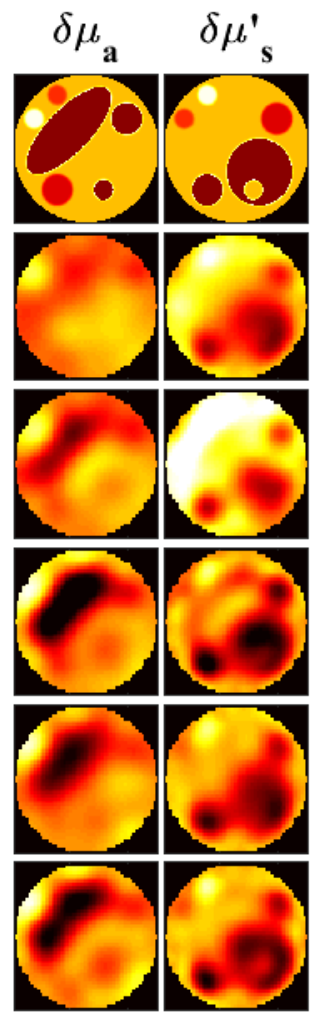

$\mu_{\mathrm{a}}=0.01$

$\mu_{\mathrm{s}}^{\prime}=1$
Target 4
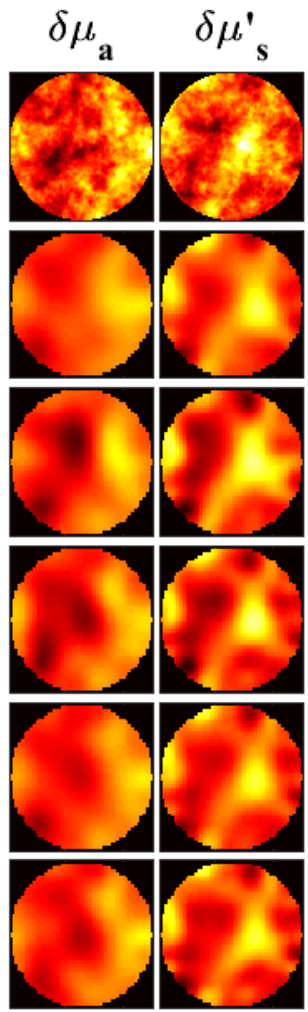

Fig. 4. Absorption $\delta \mu_{\mathrm{a}}\left(\mathrm{mm}^{-1}\right)$ and scattering $\delta \mu_{\mathrm{s}}^{\prime}\left(\mathrm{mm}^{-1}\right)$ difference distributions. First row: target distribution. From second to sixth rows: estimated parameters obtained using data types mean time of flight $\langle t\rangle$, variance $c_{2}$, their combination $\left.(<t\rangle, c_{2}\right)$, and Fourier transformed data at one $\mathrm{FT}_{\mathrm{N} \omega=1}$ and five $\mathrm{FT}_{\mathrm{N} \omega=5}$ frequencies.

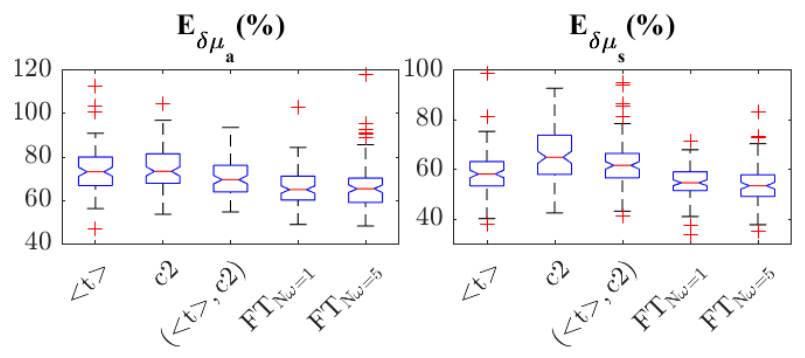

Fig. 5. Statistics of the relative errors $E_{\delta \mu_{\mathrm{a}}}(\%)$ and $E_{\delta \mu_{\mathrm{s}}^{\prime}}(\%)$ of the absorption and scattering difference estimates from 100 target samples. The utilised data types were the mean time of flight $\langle t\rangle$, variance $c_{2}$, their combination $\left(\langle t\rangle, c_{2}\right)$, and Fourier transformed data at one $\mathrm{FT}_{\mathrm{N}_{\omega}=1}$ and five $\mathrm{FT}_{\mathrm{N}_{\omega}=5}$ frequencies.

In this work, we did not consider transformation of noise from the time-resolved measurement data to temporal moments or frequency domain. Rather, we added noise to the transformed data itself. Hence, the results presented here are applicable in cases where the noise in each data type is well known. Estimating noise in the transformed data types is a challenging problem in itself and beyond the scope of this paper.

Furthermore, in this work, we studied DOT with target op-
Table 4. Computation times for absolute $T_{\mathrm{abs}}(s)$ and difference $T_{\text {diff }}(s)$ imaging, and the number of iterations $N_{\text {iter }}$ required to reach the convergence of the Gauss-Newton algorithm. The utilised data types were the mean time of flight $\langle t\rangle$, variance $c_{2}$, their combination $\left(\langle t\rangle, c_{2}\right)$, and Fourier transformed data at one $\mathbf{F T}_{\mathrm{N}_{\omega}=1}$ and five $\mathbf{F T}_{\mathrm{N}_{\omega}=5}$ frequencies.

\begin{tabular}{cccccc}
\hline & $<t>$ & $c_{2}$ & $\left(<t>, c_{2}\right)$ & $\mathrm{FT}_{\mathrm{N}_{\omega}=1}$ & $\mathrm{FT}_{\mathrm{N}_{\omega}=5}$ \\
\hline$T_{\text {diff }}$ & 11 & 26 & 35 & 3 & 7 \\
$T_{\text {abs }}$ & 49 & 187 & 162 & 8 & 52 \\
$N_{\text {iter }}$ & 6 & 8 & 5 & 5 & 5 \\
\hline
\end{tabular}

tical parameters in diffuse regime. With smaller size targets or in a medium with low-scattering regions, the DA is not a valid approximation and the radiative transfer equation need to be utilised. In this case, the most suitable data types and the number of frequencies in the Fourier transformed data may be different, and would require a separate study. For a study utilising truncated Fourier-series to approximate the solution of the time-domain radiative transfer equation, see [43].

Overall, the results show that utilising temporal moments and Fourier transformed data in TD-DOT results in good quality 
reconstructions with good estimation accuracy. Furthermore, these estimates are improved if more than one data type is used. Computationally, it seems that the frequency domain computations are beneficial to the temporal moments due to the more simple computation of Jacobians. The future work includes, in addition to above mentioned, evaluation of these techniques with experimental data.

\section{APPENDIX A: CALCULATION OF JACOBIANS}

In order to form Jacobian matrices for the Gauss-Newton algorithm, derivatives of the FE-matrices used to numerically approximate the solution of forward models need to be computed. These are obtained as follows. The FE-sensitivity matrices corresponding to the mean time of flight $<t>$ are given by

$$
\frac{\partial<\mathbf{t}>}{\partial \mu}=\frac{\left(\begin{array}{c}
\mathbf{m}_{\mathbf{0}}\left(-\mathcal{M} \mathbf{K}^{-1} \frac{\partial \mathbf{K}}{\partial \mu} \mathbf{K}^{-1}\left(\mathbf{M}\left(\mathbf{K}^{-1} \mathbf{Q}\right)\right)\right. \\
\left.-\mathcal{M} \mathbf{K}^{-1}\left(\mathbf{M}\left(\mathbf{K}^{-1} \frac{\partial \mathbf{K}}{\partial \mu} \mathbf{K}^{-1} \mathbf{Q}\right)\right)\right) \\
+\mathbf{m}_{\mathbf{1}}\left(\mathcal{M} \mathbf{K}^{-1} \frac{\partial \mathbf{K}}{\partial \mu} \mathbf{K}^{-1} \mathbf{Q}\right)
\end{array}\right)}{\mathbf{m}_{\mathbf{0}}{ }^{2}}
$$

where $\mu$ is either $\mu_{\mathrm{a}}$ or $\mu_{\mathrm{s}}^{\prime}$. Further, $\frac{\partial \mathbf{K}}{\partial \mu}$ for $\mu=\mu_{\mathrm{a}}$ and $\mu=\mu_{\mathrm{s}}^{\prime}$ are

$$
\begin{aligned}
\frac{\partial \mathbf{K}}{\partial \mu_{\mathrm{a}}}= & \int_{\Omega} \sum_{l=1}^{\mathrm{N}_{\mathrm{n}}}\left(\frac{1}{\left(d\left(\mu_{\mathrm{a}, l}+\mu_{\mathrm{s}, l}^{\prime}\right)\right)^{2}} \psi_{l}(r) \nabla \psi_{m}(r) \cdot \nabla \psi_{k}(r)\right) \mathrm{d} r \\
& +\int_{\Omega} \sum_{l=1}^{\mathrm{N}_{\mathrm{n}}} \psi_{l}(r) \psi_{m}(r) \nabla \psi_{k}(r) \mathrm{d} r \\
\frac{\partial \mathbf{K}}{\partial \mu_{\mathrm{s}}^{\prime}}= & \int_{\Omega} \sum_{l=1}^{\mathrm{N}_{\mathrm{n}}}\left(\frac{1}{\left(d\left(\mu_{\mathrm{a}, l}+\mu_{\mathrm{s}, l}^{\prime}\right)\right)^{2}} \psi_{l}(r) \nabla \psi_{m}(r) \cdot \nabla \psi_{k}(r)\right) \mathrm{d} r .
\end{aligned}
$$

The FE-sensitivity matrices for the second central moment $c_{2}$ are given by

$$
\frac{\partial \mathbf{c}_{2}}{\partial \mu}=\frac{\left(\begin{array}{r}
2 \mathbf{m}_{\mathbf{0}}\left(-\mathcal{M} \mathbf{K}^{-1} \frac{\partial \mathbf{K}}{\partial \mu} \mathbf{K}^{-1}\left(\mathbf{M}\left(\mathbf{K}^{-1} \mathbf{M}\left(\mathbf{K}^{-1} \mathbf{Q}\right)\right)\right)\right. \\
-\mathcal{M} \mathbf{K}^{-1}\left(\mathbf{M} \mathbf{K}^{-1} \frac{\partial \mathbf{K}}{\partial \mu} \mathbf{K}^{-1}\left(\mathbf{M}\left(\mathbf{K}^{-1} \mathbf{Q}\right)\right)\right) \\
\left.-\mathcal{M} \mathbf{K}^{-1}\left(\mathbf{M} \mathbf{K}^{-1}\left(\mathbf{M} \mathbf{K}^{-1} \frac{\partial \mathbf{K}}{\partial \mu} \mathbf{K}^{-1} \mathbf{Q}\right)\right)\right) \\
+\mathbf{m}_{\mathbf{2}}\left(\mathcal{M} \mathbf{K}^{-1} \frac{\partial \mathbf{K}}{\partial \mu} \mathbf{K}^{-1} \mathbf{Q}\right)
\end{array}\right)}{\mathbf{m}_{\mathbf{0}}^{2}}
$$

Furthermore, the FE-sensitivity matrices for logarithm of amplitude and phase of the frequency domain exitance at the frequency $\omega_{k}$ can be computed as

$$
\frac{\partial}{\partial \mu}\left(\begin{array}{l}
\operatorname{Re} \\
\operatorname{Im}
\end{array}\right) \log \boldsymbol{\Gamma}\left(\omega_{k}\right)=\left(\begin{array}{l}
\operatorname{Re} \\
\operatorname{Im}
\end{array}\right) \frac{-\mathcal{M}\left[\mathbf{K}+\frac{\mathrm{i} \omega_{k}}{\mathrm{c}} \mathbf{M}\right]^{-1} \frac{\partial \mathbf{K}}{\partial \mu} \boldsymbol{\Phi}\left(\omega_{k}\right)}{\Gamma\left(\omega_{k}\right)} .
$$

\section{APPENDIX B: UTILISING REAL AND IMAGINARY DATA}

In a previous work [24], we utilised the real and imaginary parts of the Fourier transformed data for absolute imaging using one
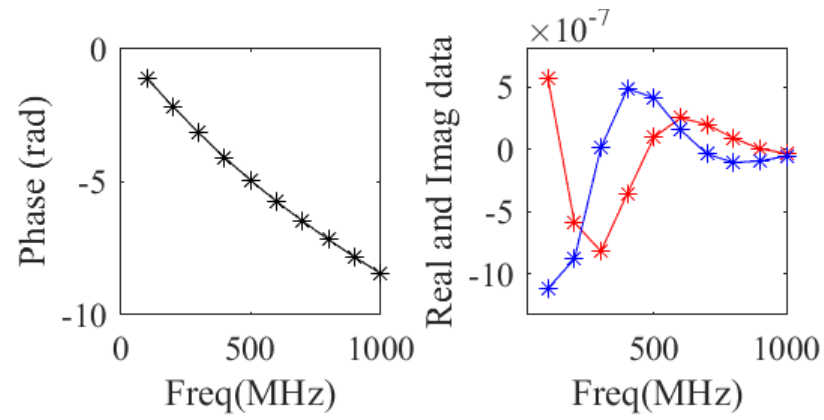

Fig. 6. Phase delay of frequency domain data at several frequencies (left image) and the corresponding real (red line) and imaginary (blue line) data (right image).

and several frequencies. We note here the difficulties with this data type compared to the logarithm of amplitude and phase, Eq. (33). The real and imaginary parts of the Fourier transformed data is given by

$$
\left(\begin{array}{l}
\operatorname{Re} \\
\operatorname{Im}
\end{array}\right) y_{\omega_{k}}=\left(\begin{array}{c}
\mathcal{A}_{\omega_{k}} \cos \phi_{\omega_{k}} \\
\mathcal{A}_{\omega_{k}} \sin \phi_{\omega_{k}}
\end{array}\right)
$$

where $\mathcal{A}_{\omega_{k}}$ is the amplitude and $\phi_{\omega_{k}}$ is the phase delay of the Fourier transformed data at frequency $\omega_{k}$.

In Fig. 6, the phase delays from frequency domain data at several frequencies is visualised together with the corresponding real and imaginary data. The data was simulated in a 2D circular domain of radius $25 \mathrm{~mm}$ with constant optical properties $\mu_{\mathrm{a}}=0.01 \mathrm{~mm}^{-1}$ and $\mu_{\mathrm{s}}^{\prime}=1 \mathrm{~mm}^{-1}$. The source and detector were positioned on the opposite sides of the domain. As shown, with higher phase delays, the corresponding real and imaginary data show cyclic behaviour due to the sine and cosine functions of the phase, Eq. (A6). Such high phase delays can be obtained in measurements from medium with high scattering parameter values. The logarithm of amplitude and phase, however, are non-cyclic and can still be utilised in such situations.

Funding. The Finnish Cultural Foundation (00200746); Jane and Aatos Erkko Foundation; Academy of Finland (314411, 312342, 336799, 320166).

Disclosures. The authors declare no conflicts of interest.

\section{REFERENCES}

1. S. R. Arridge, "Optical tomography in medical imaging," Inverse Probl. 15, R41 (1999).

2. A. Gibson, J. Hebden, and S. R. Arridge, "Recent advances in diffuse optical imaging," Phys. Medicine \& Biol. 50, R1 (2005).

3. T. Durduran, R. Choe, W. B. Baker, and A. G. Yodh, "Diffuse optics for tissue monitoring and tomography," Rep. Prog. Phys. 73, 076701 (2015).

4. C. Darne, Y. Lu, and E. Sevick-Muraca, "Small animal fluorescence and bioluminescence tomography: a review of approaches, algorithms and technology update," Phys. Med. Biol. 59, R1-R64 (2014).

5. D. Grosenick, H. Rinneberg, R. Cubeddu, and P. Taroni, "Review of optical breast imaging and spectroscopy," J. Biomed. Opt. 21, 091311 (2016).

6. Y. Hoshi and Y. Yamada, "Overview of diffuse optical tomography and its clinical applications," J. Biomed. Opt. 21, 091312 (2016). 
7. A. Pifferi, D. Contini, A. Dalla Mora, A. Farina, L. Spinelli, and A. Torricelli, "New frontiers in time-domain diffuse optics, a review," J. Biomed. Opt. 21, 091310 (2016)

8. H. Eda, I. Oda, Y. Ito, Y. Wada, Y. Oikawa, Y. Tsunazawa, M. Takada, Y. Tsuchiya, Y. Yamashita, M. Oda, A. Sassaroli, Y. Yamada, and M. Tamura, "Multichannel time-resolved optical tomographic imaging system," Rev. Sci. Instruments 70, 3595-3602 (1999).

9. A. Farina, M. Betcke, L. Di Sieno, A. Bassi, N. Ducros, A. Pifferi, G. Valentini, S. Arridge, and C. D'Andrea, "Multiple-view diffuse optical tomography system based on time-domain compressive measurements," Opt. Lett. 42, 2822-2825 (2017).

10. F. E. Schmidt, M. E. Fry, E. M. Hillman, J. C. Hebden, and D. T. Delpy, "A 32-channel time-resolved instrument for medical optical tomography," Rev. Sci. Instruments 71, 256-265 (2000)

11. B. Montcel, R. Chabrier, and P. Poulet, "Detection of cortical activation with time-resolved diffuse optical methods," Appl. Opt. 44, 1942-1947 (2005).

12. J. J. Selb, D. K. Joseph, and D. A. Boas, "Time-gated optical system for depth-resolved functional brain imaging," J. Biomed. Opt. 11, 044008 (2006).

13. D. Milej, A. Gerega, M. Kacprzak, P. Sawosz, W. Weigl, R. Maniewski, and A. Liebert, "Time-resolved multi-channel optical system for assessment of brain oxygenation and perfusion by monitoring of diffuse reflectance and fluorescence," Opto-Electronics Rev. 22, 55-67 (2014).

14. L. C. Enfield, A. P. Gibson, J. C. Hebden, and M. Douek, "Optical tomography of breast cancer-monitoring response to primary medical therapy," Target. Oncol. 4, 219-233 (2009).

15. J. M. Cochran, D. R. Busch, L. Lin, D. L. Minkoff, M. Schweiger, S. Arridge, and A. G. Yodh, "Hybrid time-domain and continuous-wave diffuse optical tomography instrument with concurrent, clinical magnetic resonance imaging for breast cancer imaging," J. Biomed. Opt. 24, 051409 (2019).

16. F. Gao, H. Zhao, and Y. Yamada, "Improvement of image quality in diffuse optical tomography by use of full time-resolved data," Appl. Opt. 41, 778-791 (2002).

17. M. A. Naser and M. Deen, "Time-domain diffuse optical tomography using recursive direct method of calculating jacobian at selected temporal points," Biomed. Phys. \& Eng. Express 1, 045207 (2015).

18. S. R. Arridge, "Photon-measurement density functions. part I: Analytical forms," Appl. Opt. 34, 7395-7409 (1995).

19. S. R. Arridge and M. Schweiger, "Photon-measurement density functions. part 2: Finite-element-method calculations," Appl. Opt. 34, 80268037 (1995)

20. S. R. Arridge and M. Schweiger, "Direct calculation of the moments of the distribution of photon time of flight in tissue with a finite-element method," Appl. Opt. 34, 2683-2687 (1995).

21. M. Schweiger and S. R. Arridge, "Application of temporal filters to time resolved data in optical tomography," Phys. Medicine \& Biol. 44, 1699 (1999).

22. S. Okawa, Y. Hoshi, and Y. Yamada, "Improvement of image quality of time-domain diffuse optical tomography with Ip sparsity regularization," Biomed. Opt. Express 2, 3334-3348 (2011).

23. A. Gibson, T. Austin, N. Everdell, M. Schweiger, S. Arridge, J. Meek, J. Wyatt, D. Delpy, and J. Hebden, "Three-dimensional whole-head optical tomography of passive motor evoked responses in the neonate," Neuroimage 30, 521-528 (2006).

24. M. Mozumder and T. Tarvainen, "Time-domain diffuse optical tomography utilizing truncated fourier series approximation," JOSA A $\mathbf{3 7}$, 182-191 (2020).

25. S. Arridge, "Methods in diffuse optical imaging," Phil. Trans. R. Soc. A 369, 4558-4576 (2011).

26. L. Hervé, A. Puszka, A. Planat-Chrétien, and J.-M. Dinten, "Timedomain diffuse optical tomography processing by using the mellinlaplace transform," Appl. Opt. 51, 5978-5988 (2012).

27. E. M. Hillman, J. C. Hebden, F. E. Schmidt, S. R. Arridge, M. Schweiger, H. Dehghani, and D. T. Delpy, "Calibration techniques and datatype extraction for time-resolved optical tomography," Rev. Sci. Instruments 71, 3415-3427 (2000).
28. E. M. Hillman, J. C. Hebden, M. Schweiger, H. Dehghani, F. E. Schmidt, D. T. Delpy, and S. R. Arridge, "Time resolved optical tomography of the human forearm," Phys. Med. Biol. 46, 1117 (2001).

29. J. C. Hebden, A. Gibson, R. M. Yusof, N. Everdell, E. M. Hillman, D. T. Delpy, S. R. Arridge, T. Austin, J. H. Meek, and J. S. Wyatt, "Threedimensional optical tomography of the premature infant brain," Phys. Med. Biol. 47, 4155 (2002).

30. I. T. Nissilä, J. C. Hebden, D. Jennions, J. Heino, M. Schweiger, K. M. Kotilahti, T. E. Noponen, A. P. Gibson, S. Järvenpää, L. Lipiäinen et al., "Comparison between a time-domain and a frequency-domain system for optical tomography," J. Biomed. Opt. 11, 064015 (2006).

31. T. Austin, A. Gibson, G. Branco, R. M. Yusof, S. Arridge, J. Meek, J. Wyatt, D. Delpy, and J. Hebden, "Three dimensional optical imaging of blood volume and oxygenation in the neonatal brain," Neuroimage. 31, 1426-1433 (2006).

32. J. Selb, A. M. Dale, and D. A. Boas, "Linear 3D reconstruction of time-domain diffuse optical imaging differential data: improved depth localization and lateral resolution," Opt. Express 15, 16400-16412 (2007).

33. J. Zouaoui, L. Di Sieno, L. Hervé, A. Pifferi, A. Farina, A. Dalla Mora, J. Derouard, and J.-M. Dinten, "Quantification in time-domain diffuse optical tomography using mellin-laplace transforms," Biomed. Opt. Exp. 7, 4346-4363 (2016)

34. D. Orive-Miguel, L. Hervé, L. Condat, and J. Mars, "Improving localization of deep inclusions in time-resolved diffuse optical tomography," Appl. Sci. 9, 5468 (2019).

35. S. R. Arridge, J. C. Hebden, M. Schweiger, F. E. W. Schmidt, M. E. Fry, E. M. C. Hillman, H. Dehghani, and D. T. Delby, "A method for three-dimensional time-resolved optical tomography," Int. J. Imag. Sys. Tech. 11, 2-11 (2000).

36. M. Schweiger and S. R. Arridge, "Direct calculation with a finite-element method of the Laplace transform of the distribution of photon time of flight in tissue," Appl. Opt. 36, 9042-9049 (1997).

37. A. Liebert, H. Wabnitz, J. Steinbrink, H. Obrig, M. Möller, R. Macdonald, A. Villringer, and $\mathrm{H}$. Rinneberg, "Time-resolved multidistance nearinfrared spectroscopy of the adult head: intracerebral and extracerebral absorption changes from moments of distribution of times of flight of photons," Appl. Opt. 43, 3037-3047 (2004).

38. H. Wabnitz, D. Contini, L. Spinelli, A. Torricelli, and A. Liebert, "Depthselective data analysis for time-domain fnirs: moments vs. time windows," Biomed. Opt. Express 11, 4224-4243 (2020).

39. S. R. Arridge and W. R. B. Lionheart, "Nonuniqueness in diffusionbased optical tomography," Opt. Lett. 23, 882-884 (1998).

40. T. Tarvainen, V. Kolehmainen, M. Vauhkonen, A. Vanne, A. P. Gibson, M. Schweiger, S. R. Arridge, and J. P. Kaipio, "Computational calibration method for optical tomography," Appl. Opt. 44, 1879-1888 (2005).

41. A. Ishimaru, Wave Propagation and Scattering in Random Media (Academic, Newyork, 1978).

42. S. Arridge, M. Schweiger, M. Hiraoka, and D. Delpy, "A finite element approach to modelling photon transport in tissue," Med. Phys. 20, 299309 (1993)

43. A. Pulkkinen and T. Tarvainen, "Truncated fourier-series approximation of the time-domain radiative transfer equation using finite elements," $\mathrm{J}$. Opt. Soc. Am. A 30, 470-478 (2013).

44. J. Kaipio and E. Somersalo, Statistical and Computational Inverse Problems (Springer, Newyork, 2005).

45. C. E. Rasmussen and C. K. I. Williams, Gaussian Processes for Machine Learning (the MIT Press, Cambridge, MA, 2006).

46. M. Schweiger and S. R. Arridge, "The Toast++ software suite for forward and inverse modeling in optical tomography," J. Biomed. Opt. 19, 040801 (2014) 